\title{
Experimental and modeling evidence for structural crossover in supercritical $\mathrm{CO}_{2}$
}

Cillian J. Cockrell, Oliver Dicks, Ling Wang, Kostya Trachenko, Alan K. Soper, Vadim V. Brazhkin, and Sarantos Marinakis

\section{Published version information}

Citation: CJ Cockrell et al. "Experimental and modeling evidence for structural crossover in supercritical $\mathrm{CO}_{2}$ ". Physical Review E, vol. 101, no. 5 (2020): 052109.

DOI: $\underline{10.1103 / P h y s R e v E .101 .052109 ~}$

This version is made available in accordance with publisher policies. Please cite only the published version using the reference above. This is the citation assigned by the publisher at the time of issuing the APV. Please check the publisher's website for any updates. 


\title{
Experimental and modeling evidence for structural crossover in supercritical $\mathrm{CO}_{2}$
}

\author{
Cillian J. Cockrell $\odot,{ }^{*}$ Oliver Dicks $\odot,{ }^{\dagger}$ Ling Wang $\odot,{ }^{\ddagger}$ and Kostya Trachenko $\odot^{\S}$ \\ School of Physics and Astronomy, Queen Mary University of London, Mile End Road, London E1 4NS, United Kingdom \\ Alan K. Soper $₫$
ISIS Facility, STFC Rutherford Appleton Laboratory, Harwell Campus, Didcot, Oxon OX11 0QX, United Kingdom
}

Vadim V. Brazhkin $\oplus^{\mathbb{I l}}$

Institute for High Pressure Physics, RAS, 108840 Moscow, Russia

Sarantos Marinakis $\oplus^{* *}$

School of Health, Sport and Bioscience, University of East London, Stratford Campus, Water Lane, London E15 4LZ, United Kingdom and Department of Chemistry and Biochemistry, School of Biological and Chemical Sciences, Queen Mary University of London, Joseph Priestley Building, Mile End Road, London E1 4NS, United Kingdom

(Received 3 February 2020; revised manuscript received 6 April 2020; accepted 8 April 2020; published 12 May 2020)

\begin{abstract}
The physics of supercritical states is understood to a much lesser degree compared to subcritical liquids. Carbon dioxide, in particular, has been intensely studied, yet little is known about the supercritical part of its phase diagram. Here, we combine neutron scattering experiments and molecular dynamics simulations and demonstrate the structural crossover at the Frenkel line. The crossover is seen at pressures as high as 14 times the critical pressure and is evidenced by changes of the main features of the structure factor and pair distribution functions.
\end{abstract}

DOI: 10.1103/PhysRevE.101.052109

\section{INTRODUCTION}

Supercritical fluids have unique properties that have led to a rich variety of applications [1]. Rare gases, nitrogen, $\mathrm{CO}_{2}$, and $\mathrm{H}_{2} \mathrm{O}$ are among the most common supercritical fluids. $\mathrm{CO}_{2}$, in particular, is an important greenhouse gas of Earth's atmosphere and, in its supercritical state, is the main component $(97 \%)$ in the atmosphere of Venus. Supercritical $\mathrm{CO}_{2}$ is used in a great variety of applications (see, e.g., applications in solubility, synthesis, and processing of polymers [2-4], dissolution and deposition in microdevices [5], green chemistry and solvation [6-12], green catalysis [9,13-15], extraction [16], chemical reactions [17], green nanosynthesis [18], and sustainable development including carbon capture and storage [19]). It has been widely appreciated that improving fundamental knowledge of the supercritical state is important for the reliability, scale-up, and widening of these applications (see, e.g., Refs. [1,7,9,12,15,17]).

Compared to subcritical liquids, the supercritical state is not well understood. Traditional understanding amounted to a general assertion that this state is physically homogeneous,

\footnotetext{
*c.j.cockrell@qmul.ac.uk

†o.dicks@qmul.ac.uk

${ }^{\ddagger}$ ling.wang@qmul.ac.uk

§k.trachenko@qmul.ac.uk

" alan.soper@stfc.ac.uk

Ibrazhkin@hppi.troitsk.ru

**s.marinakis@uel.ac.uk
}

with no qualitative changes taking place anywhere above the critical point [1]. The first challenge to this view was the Widom line. Close to the critical point, the Widom line characterizes persisting near-critical anomalies such as the maximum in the heat capacity [20], which can be used to stratify different states in the supercritical region. A different subsequent proposal was based on the Frenkel line (FL) separating two distinct states in the supercritical state with liquidlike and gaslike dynamics. Differently from the Widom line, the FL extends to arbitrarily high pressures and temperatures (as long as chemical bonding is unaltered), is unrelated to the critical point, and exists in systems with no boiling line or critical point [21-23]. The FL is also of practical importance because it corresponds to the solubility maxima in supercritical $\mathrm{CO}_{2}$ [24].

Here, we combine neutron scattering experiments and molecular dynamics (MD) simulations and show evidence of the structural crossover of supercritical carbon dioxide at the FL. The crossover extends to pressure as high as 14 times the critical pressure and is evidenced by changes in the main features of the structure factor (SF) and pair distribution functions (PDFs). The neutron scattering experiments evidencing a crossover at highly supercritical pressures are the first of their kind for $\mathrm{CO}_{2}$.

\section{METHODS}

We recall that particle dynamics combine solidlike oscillations around quasiequilibrium positions and diffusive jumps 
between different positions below the FL, the typical character of molecular motion in liquids [25]. Above the line, particle dynamics lose this oscillatory component and become purely diffusive. This gives a practical criterion to calculate the FL based on the disappearance of minima of the velocity autocorrelation function [22]. This criterion coincides with the thermodynamic criterion $c_{v}=2 k_{B}$ corresponding to the disappearance of transverselike excitations in a monatomic system [26]. Since structure and dynamics are related [27], the FL crossover was predicted to result in a crossover of the supercritical structure.

The pressures we consider for both experiments and MD simulations are 500 and 590 bar. The $\mathrm{FL}$ in $\mathrm{CO}_{2}$ was previously calculated using the velocity autocorrelation function criterion [24], giving us the following two state points of the predicted crossover: (500 bar, $297 \mathrm{~K}$ ) and (590 bar, $302 \mathrm{~K})$. We recall that the FL extends to arbitrarily high pressures and temperatures above the critical point, but at low temperatures it touches the boiling line at around $0.8 T_{c}$, where $T_{c}$ is the critical temperature [22]. (Note that the system does not have cohesive liquidlike states at temperatures above approximately $0.8 T_{c}$ [28], hence crossing the boiling line at around $0.8 T_{c}$ and above can be viewed as a gas-gas transition [22].) The critical point of $\mathrm{CO}_{2}$ is (73.9 bar, $\left.304.3 \mathrm{~K}\right)$, hence our state points correspond to near-critical temperatures and pressures well above critical. In this regard, we note that the supercritical state is often defined as the state at $P>P_{c}$ and $T>T_{c}$. This definition is loose, not least because an isotherm drawn on a $(P, T)$ diagram above the critical point crosses the melting line, implying that the supercritical state can be found in the solid phase. As a result, one can meaningfully speak about the near-critical part of the phase diagram only when discussing the location of the supercritical state on the phase diagram [29]. As far as our state points are concerned, they correspond to temperatures much higher than the melting temperature and pressures extending to 14 times the critical pressure where near-critical anomalies are nonexistent [29].

A cylinder of carbon dioxide was obtained from BOC, CP grade, and used without further purification. The pressure of the cylinder was around 50 bar and a SITEC intensifier and a SITEC hand-pump gas was used to raise the pressure. Capillaries were used to connect the intensifier manifold system to the cell. The flat plate pressure cell was made from an alloy of $\mathrm{Ti}$ and $\mathrm{Zr}$ at the mole ratio 0.676:0.324, which contributes almost zero coherent scattering to the diffraction pattern [30]. The cell consisted of a flat section that was $12 \mathrm{~mm}$ thick and had four 6-mm-diameter holes running through it, so the occupied gas space was $6 \mathrm{~mm}$ thick and the wall thickness was $3 \mathrm{~mm}$ on either side. The container was placed at a right angle to the neutron beam, which was approximately $30 \times 30 \mathrm{~mm}$ in cross section. A bottom-loading, closed-cycle helium refrigerator was used to control the temperature within $\pm 1 \mathrm{~K}$, using He exchange gas at $\sim 20$ mbar to provide temperature uniformity. The employed temperatures and pressures are listed in Table I, where the densities were calculated from the data available in the NIST database [31].

Total neutron scattering measurements were performed on the NIMROD diffractometer at the ISIS pulsed neutron source [32]. Absolute values of the differential cross sections were obtained from the raw scattering data by normalizing the data
TABLE I. $T-P-d$ state points for neutron scattering measurements. Values of $d$ are taken from [31].

\begin{tabular}{lcccc}
\hline \hline $\begin{array}{c}T_{\exp } \\
(\mathrm{K})\end{array}$ & $\begin{array}{c}P_{\exp } \\
\text { (bar) }\end{array}$ & $\begin{array}{c}d \\
(\mathrm{~g} / \mathrm{mL})\end{array}$ & $\begin{array}{c}P_{\exp } \\
(\text { bar })\end{array}$ & $\begin{array}{c}d \\
(\mathrm{~g} / \mathrm{mL})\end{array}$ \\
\hline 250 & 500 & 1.1676 & 590 & 1.1821 \\
270 & 500 & 1.1131 & 590 & 1.1306 \\
290 & 500 & 1.0573 & 590 & 1.0784 \\
310 & 500 & 1.0003 & 590 & 1.0257 \\
330 & 500 & 0.9426 & 590 & 0.9729 \\
340 & 500 & 0.9137 & - & - \\
350 & 500 & 0.8848 & 590 & 0.9204 \\
360 & 500 & 0.8560 & - & - \\
370 & 500 & 0.8276 & 590 & 0.8688 \\
380 & 500 & 0.7996 & 590 & 0.8436 \\
390 & 500 & 0.7722 & 590 & 0.8188 \\
\hline \hline
\end{tabular}

to the scattering from a slab of vanadium of known thickness and were further corrected for background and multiple scattering, container scattering, and self-attenuation, using the Gudrun data analysis program [33]. Finally, the data were put on an absolute scale of barns (b) per atom per sr by dividing by the number of atoms in the neutron beam $\left(1 \mathrm{~b}=10^{-28} \mathrm{~m}^{2}\right)$,

$$
F(Q)=\frac{1}{9} b_{\mathrm{C}}^{2} H_{\mathrm{CC}}(Q)+\frac{4}{9} b_{\mathrm{O}}^{2} H_{\mathrm{OO}}(Q)+\frac{4}{9} b_{\mathrm{C}} b_{\mathrm{O}} H_{\mathrm{CO}}(Q),
$$

where $b_{\alpha}$ is the neutron scattering length of atom $\alpha$, the partial SF $H_{\alpha \beta}(Q)$ is the three-dimensional Fourier transform of the corresponding site-site radial distribution function,

$$
H_{\alpha \beta}(Q)=4 \pi \rho \int_{0}^{\infty} r^{2}\left(g_{\alpha \beta}(r)-1\right) \frac{\sin Q r}{Q r} d r
$$

and $\rho$ is the atomic number density. Note that the $H_{\mathrm{OO}}(Q)$ and $H_{\mathrm{CO}}(Q)$ terms include both the intra- and the intermolecular scattering. The results are shown in Fig. 1.

The MD simulation package DL_POLY [34] was used to simulate a system of $30752 \mathrm{CO}_{2}$ particles with periodic boundary conditions. The potential for $\mathrm{CO}_{2}$ is a rigid-body nonpolarizable potential based on a quantum chemistry calculation, with the partial charges derived using the distributed multipole analysis method [35]. The electrostatic interactions were evaluated using the smooth particle mesh Ewald method in MD simulations. The potential was derived and tuned using a large suite of energies from $a b$ initio density functional theory calculations of different molecular clusters and validated against various sets of experimental data including phonon dispersion curves and $P V T$ data. These data included solid, liquid, and gas states and gas-liquid coexistence lines and extended to high-pressure and high-temperature conditions [35]. We also used another rigid-body nonpolarizable potential developed by Zhang and Duan [36] and found the same results.

The MD systems were first equilibrated in the constantpressure and -temperature ensemble for $500 \mathrm{ps}$. The data were subsequently collected from production runs in the constantenergy and -volume ensemble. In order to reduce noise and see the crossover clearly, data were averaged over 500000 frames, involving production runs of a further 500 ps. 

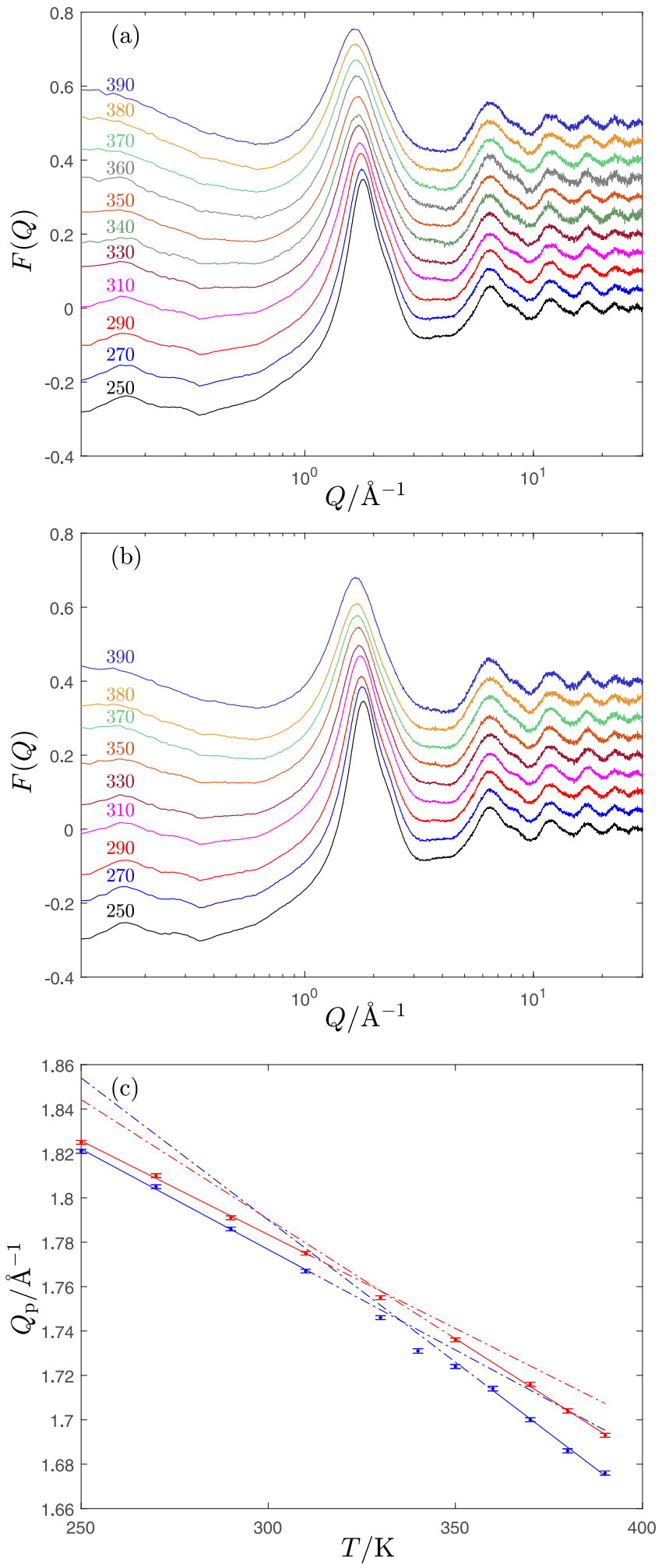

FIG. 1. Weighted sum of the experimental weighted partial structure factors $F(Q)$ for $\mathrm{CO}_{2}$ at (a) 500 bar and (b) 590 bar. Curves for higher temperatures have been shifted along the $y$ axis by 0.05 per set. (c) Position of the maximum of the first peak in the total (weighted sum) experimental structure factor as a function of the temperature for $\mathrm{CO}_{2}$ at 500 bar (blue lines) and 590 bar (red lines). Straight lines are visual guides.

\section{RESULTS AND DISCUSSION}

Before analyzing the data, we recall that the FL corresponds to the qualitative change in particle dynamics: from combined solidlike oscillatory and diffusive dynamics below the line to purely diffusive gaslike dynamics above the line. Therefore, the supercritical structure is predicted to show the crossover between the liquidlike and the gaslike structural correlations. This is predicted to be the case for functions characterizing the structure, such as the pair distribution function and structure factor. In our analysis, we focus on meaningful features such as maximum positions of PDFs and SFs.

The experimental weighted sum of the partial SFs are plotted in Fig. 1 for two pressures. We plot the first peak positions of SFs vs the temperature in Fig. 1 and observe that it undergoes the crossover at temperatures close to $320 \mathrm{~K}$ and around $12 \%$ larger than the FL crossover temperature predicted from the velocity autocorrelation function criterion mentioned earlier.

The SFs were Fourier transformed to obtain the experimental PDF. As with previous experimental and modeling results on $\mathrm{Ar}$ [27], $\mathrm{Ne}$ [37], $\mathrm{CH}_{4}$ [38], and, especially, $\mathrm{H}_{2} \mathrm{O}$ [39], pronounced changes in the first peak position in the PDF with temperature are observed, indicating a well-defined crossover. When a system is compressed or expanded, one expects

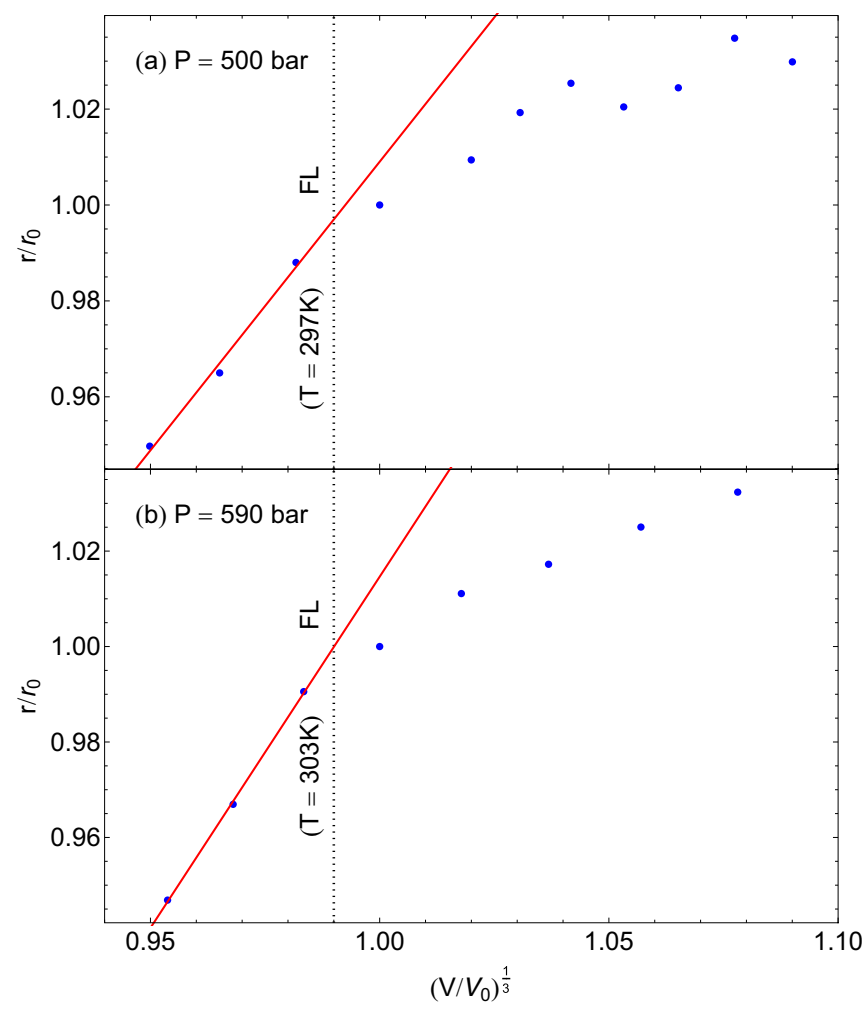

FIG. 2. First peak positions of the experimental weighted sum PDF for $\mathrm{CO}_{2}$ at (a) 500 bar and (b) 590 bar as a function of the volume. Dashed vertical lines show the reduced volumes at the FL, and straight lines are visual guides. 


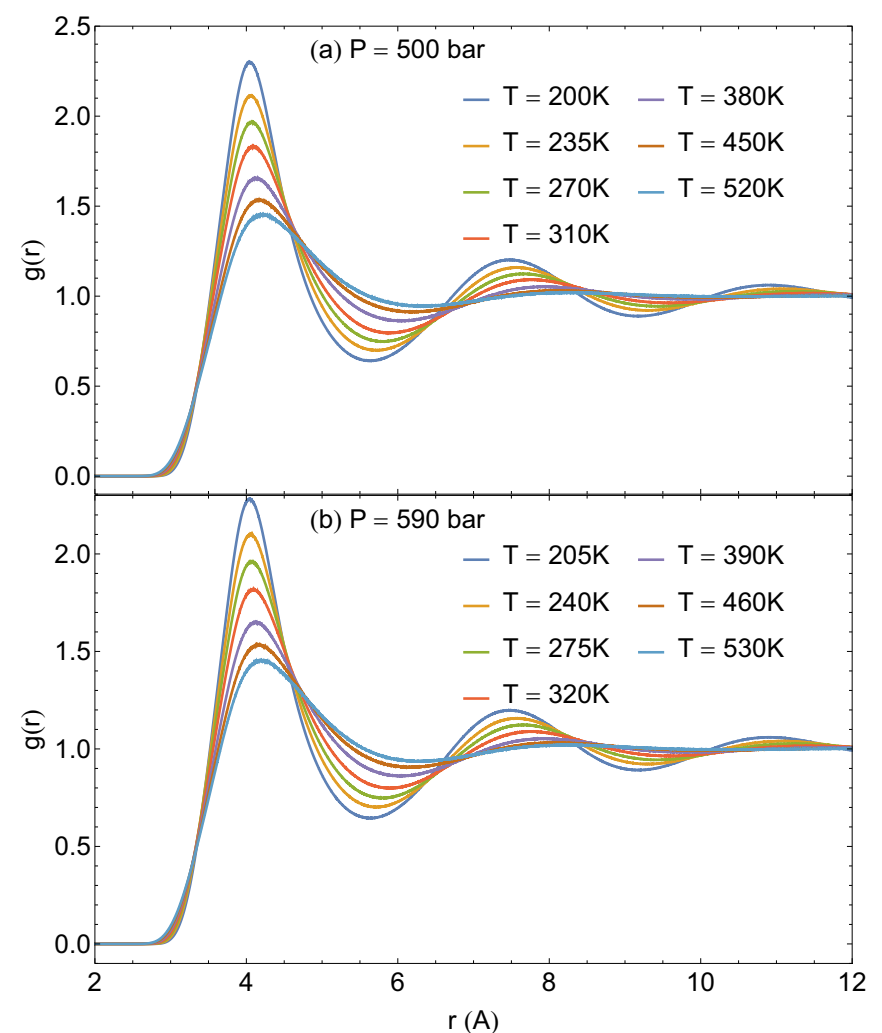

FIG. 3. Evolution of the simulated C-C pair distribution functions with temperature at (a) 500 bar and (b) 590 bar.

the first nearest-neighbor distance, $r_{\text {fnn }}$ [given by the radial position of the first peak in $g(r)$ ], and the system's "length" $\left(V^{1 / 3}\right)$ to be proportional to each other unless the system undergoes a structural change. In other words the system structure undergoes uniform compression. The first PDF peak position divided by the position $\left(r_{0}\right)$ at the Frenkel temperature vs the cube root of the volume divided by the volume $\left(V_{0}\right)$ at the same reference temperature is shown in Fig. 2. For a system undergoing uniform compression, $V / V_{0}$ and $r / r_{0}$ will be equal. If there is a phase transition at higher densities, as there is in liquids across the melting line, this rule cannot be extrapolated down to arbitrarily low volumes and hence there will be an intercept, $V^{\frac{1}{3}}=\alpha r+\beta$, upon which the gradient of $V / V_{0}$ vs $r / r_{0}$ will depend. However, as long as no structural changes occur, the gradient will remain constant. Specifically, in a simple cubic crystalline solid (atomic packing fraction, $0.52)$ the constant of proportionality between $V / N$ and $r$ is unity, in an FCC lattice (packing fraction, 0.74) the constant is 0.89 , and in a diamond cubic lattice (packing fraction, 0.34 ) the constant is 1.2. In gases, the fnn distance is largely determined by the size, geometry, and interaction of the constituent molecules (see, e.g., [40]) rather than the density. This linear relationship has been experimentally observed in molten group 1 elements [41,42] and liquid $\mathrm{CS}_{2}$ [43]. The fnn distance is most readily extracted from the partial C-C PDFs. The experimental data give the total PDF, but the peak corresponding to the fnn distance is not profoundly changed, therefore the total PDF gives a qualitative approximation of the fnn distance. In Fig. 2 we observe the crossover of the first PDF peak at a temperature within $10 \%$ of the predicted
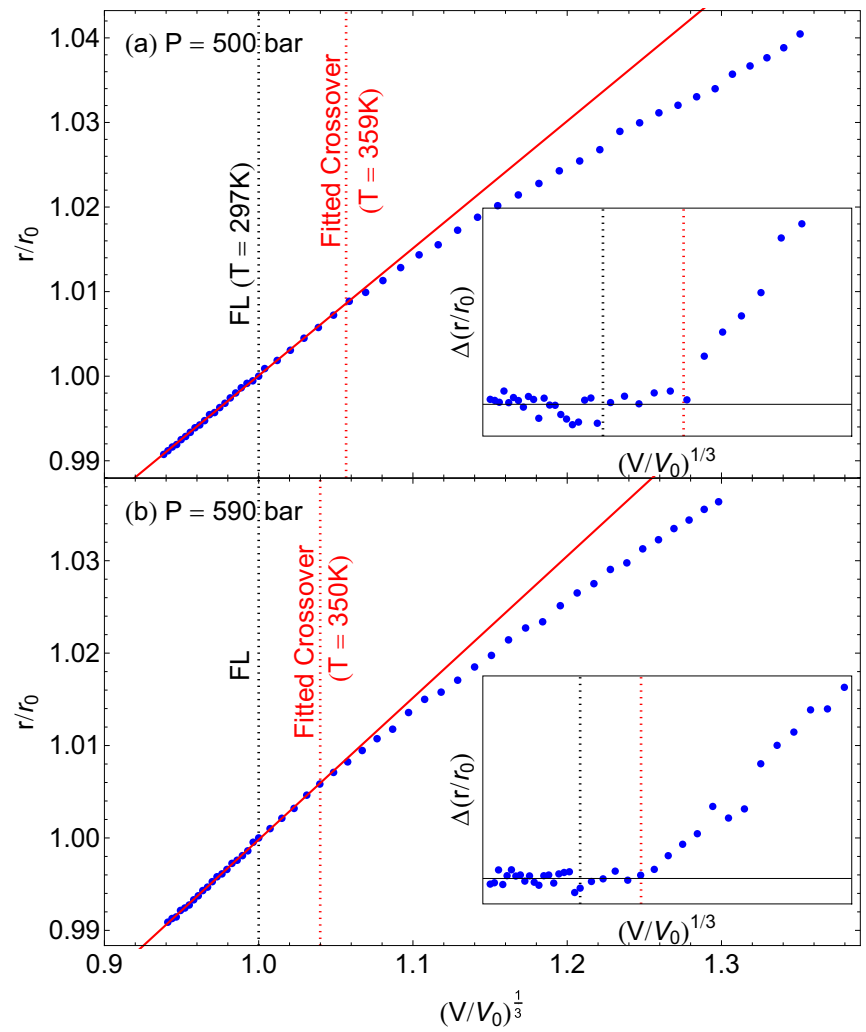

FIG. 4. First peak position of simulated C-C PDF. Straight lines are fitted to data below the FL and serve as visual guides. Dashed vertical lines show the fitted crossover volume and the volume at the FL. Insets: Relative trend of the residuals of the linear fit.

crossover temperature at the FL, signified by the change of gradients. This qualitative behavior is seen more clearly in the MD results (Fig. 4).

We now discuss the MD results. Examples of C-C PDFs from MD simulations are shown in Fig. 3. We observe a reduction in height and a corresponding broadening of peaks with increasing temperature as expected. The steepness of the first peak is related to the softness of the effective intermolecular potential, and its reduction can be quantitatively related to the reduction in the viscosity [44]. Figure 4 displays the radial positions of the first PDF peaks as a function of the volume, as discussed above, which shows a crossover at densities near the FL. Because of the reduced noise and abundance of temperature points we can perform a statistical analysis of the data to quantify the crossover. We see the same behavior, including a much clearer crossover, for both pressures. The constant of proportionality between $V / N$ and $r$ is $\approx 3.4$, implying a much more open arrangement than the crystal systems quoted above. This is in accordance with the density of $\mathrm{CO}_{2}$ at the FL $\left(23346 \mathrm{~mol} / \mathrm{m}^{3}\right)$, less than half that of water $\left(56501 \mathrm{~mol} / \mathrm{m}^{3}\right)$ at the same pressure and temperature. In order to quantify the crossover, we fitted the data to two types of functions. The first was a single functional dependence over the entire range. In order to avoid the extrapolation errors associated with high-order polynomials, the trial functions we used were quadratic or log plus linear: $f(x)=a+b x+c \log (x)$, with $a, b$, and $c$ the fitting parameters. The second set of functions was linear below a certain 
crossover volume $V_{c}$ and either quadratic or log plus linear above that volume (i.e., a piecewise function): $f(x)=\Theta\left(V_{c}-\right.$ $V)[a+b x]+\Theta\left(V-V_{c}\right)[\alpha+\beta x+\gamma \log (x)]$, with $\Theta[V]$ the Heaviside step function and $a, b, \beta, \gamma$, and $V_{c}$ the fitting parameters $(\alpha$ depends on the other parameters in order to ensure continuity of the function).

Generally speaking, adding more parameters to a fitting function improves the numerical quality of the fit. A priori, one can penalize having too many parameters; this prevents the extreme situation of a perfect fit acquired using a piecewise function with a number of subdomains equal to the number of data. The two closely related quantitative measures of goodness of fit with penalty terms for the number of parameters are the Akaike information criterion [45] and the Bayesian information criterion [46]. Applied to our data, at both pressures and with the quadratic and $\log$ plus linear variants, the Akaike and Bayesian information criteria were substantially lower than -10 below those for the single function, representing a decisive preference for two different functional dependences above and below a certain volume $\left(V_{c}\right)$. This volume is shown by the dotted vertical line (Fig. 4) and corresponds at both pressures to a temperature close to $350 \mathrm{~K}$, which is within $12 \%-15 \%$ of the predicted crossover value. Also plotted as insets in Fig. 4 are the residuals of the low-volume linear fits, which show a sharp and sudden increase above the crossover volume, which would not be the case if we had simply interpolated a straight line between nonlinear data.

Figure 5 shows theoretical PDF peak heights. We note that the PDF peak heights of a solid, $h=g\left(r_{\text {peak }}\right)-1$, are predicted $[25,47]$ to have a power-law relationship with the temperature, resulting in the relation $\log h \propto-\log T$ with $h=g(r)-1$ at the peak. The same relation can be argued to apply to liquids below the FL where the solidlike oscillatory component of molecular motion is present [27]. This is because for small displacements the energy is roughly quadratic and the displacement distribution will be Gaussian. The height of a Gaussian distribution follows a power-law relationship with its variance and, thus, with the temperature. The peak heights in Fig. 5 clearly show the crossover at the FL, with the observed crossover temperatures differing from the predicted ones by about $7 \%-15 \%$. This is in agreement with the width of the FL crossover seen experimentally and modeled on the basis of structural and thermodynamic properties $[37,48]$.

Before concluding, we note that previous experiments detecting the structural crossover at the FL involved $x$-ray scattering in supercritical $\mathrm{Ne}$ [37], the combination of x-rays with Raman scattering in supercritical $\mathrm{CH}_{4}$ [38], and the combination of neutron and Raman scattering in supercritical $\mathrm{N}_{2}$ [49] . Only one small-angle neutron scattering experiment had been used to study the $\mathrm{FL}$ in $\mathrm{CO}_{2}$ in the vicinity of the critical point only [50]. Our current neutron scattering experiment detecting the crossover at the FL at highly supercritical pressures is the first of its kind and importantly widens the range of techniques used to detect the FL. It will stimulate further

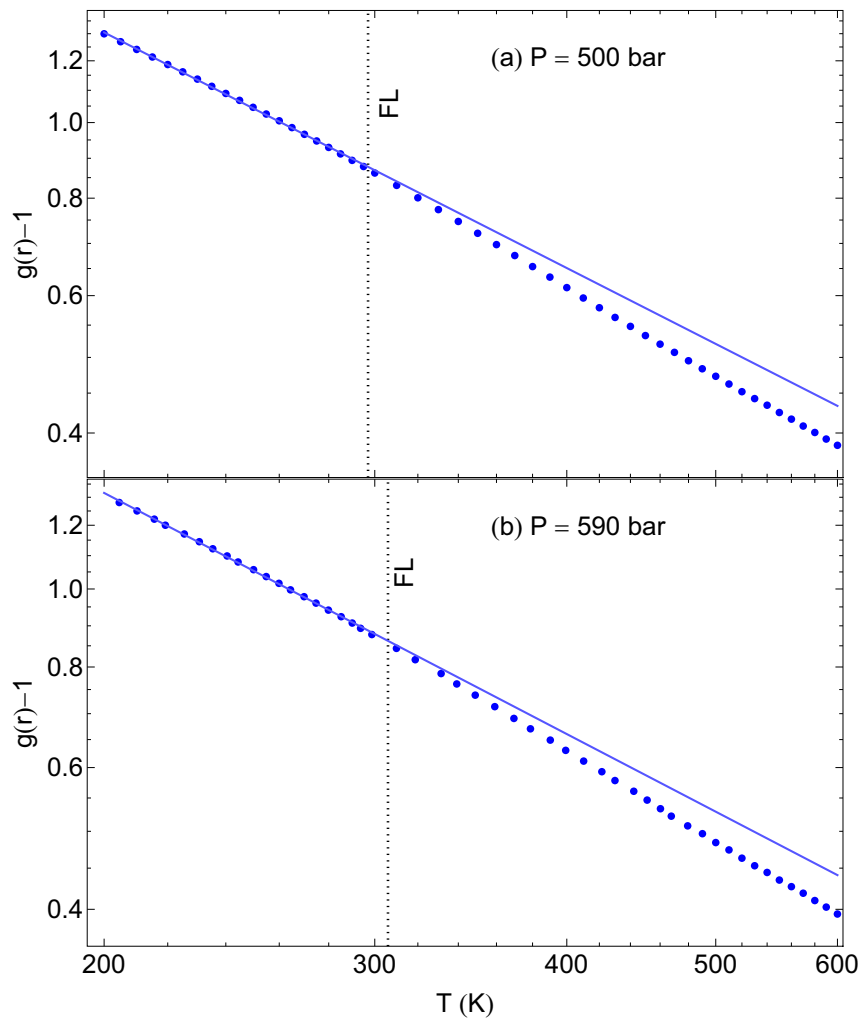

FIG. 5. First peak height of the C-C PDF for simulated $\mathrm{CO}_{2}$ at (a) 500 bar and (b) 590 bar as a function of the volume. Dashed vertical lines show the temperatures of the predicted crossover, and solid lines show the fitted straight lines.

neutron scattering experiments in important systems such as supercritical $\mathrm{H}_{2} \mathrm{O}$ where a pronounced crossover at the FL was recently predicted on the basis of MD simulations [39].

In summary, our combined neutron scattering and $\mathrm{MD}$ simulations study has detected the structural crossover in $\mathrm{CO}_{2}$ at pressures well above the critical pressure and temperatures well in excess of the melting temperature. The crossover is seen in the main features of the SF and PDFs and corresponds to the predicted crossover at the FL. Apart from the fundamental importance of understanding the supercritical state, the FL corresponds to the solubility maxima of several solutes in supercritical $\mathrm{CO}_{2}[24]$ and is therefore of practical importance.

\section{ACKNOWLEDGMENTS}

We acknowledge Chris Goodway, Thomas Headen, and Damian Fornalski (Rutherford Appleton Laboratory) for help with the experiments. Neutron beam time at ISIS and project funding were provided by the Science and Technology Facilities Council (RB1720056). This research utilized Queen Mary's MidPlus computational facilities, supported by QMUL Research-IT [51].
[1] E. Kiran, P. G. Debenedetti, and C. J. Peters, Supercritical Fluids: Fundamentals and Applications, NATO Sci- ence Series E: Applied Sciences, Vol. 366 (Kluwer, Boston, 2000). 
[2] T. Sarbu, T. Styranec, and E. J. Beckman, Nature 405, 165 (2000).

[3] J. M. DeSimone, Z. Guan, and C. S. Elsbernd, Science 257, 945 (1992).

[4] A. I. Cooper, J. Mater. Chem. 10, 207 (2000).

[5] J. M. Blackburn, D. P. Long, A. Cabañas, and J. J. Watkins, Science 294, 141 (2001).

[6] J. M. DeSimone, Science 297, 799 (2002).

[7] C. A. Eckert, B. L. Knutson, and P. G. Debenedetti, Nature 383, 313 (1996).

[8] C. J. Li and B. M. Trost, Proc. Natl. Acad. Sci. U.S.A. 105, 13197 (2008).

[9] W. Leitner, Acc. Chem. Res. 35, 746 (2002).

[10] P. T. Anastas and M. M. Kirchhoff, Acc. Chem. Res. 35, 686 (2002).

[11] P. Anastas and N. Eghbali, Chem. Soc. Rev. 39, 301 (2010).

[12] E. J. Beckman, J. Super. Fluids 28, 121 (2004).

[13] D. J. Cole-Hamilton, Science 299, 1702 (2003).

[14] P. G. Jessop, Y. Hsiao, T. Ikarita, and R. Noyori, J. Am. Chem. Soc. 118, 344 (1996).

[15] P. G. Jessop, T. Ikariya, and R. Noyori, Chem. Rev. 99, 475 (1999).

[16] E. Reverchon, J. Super. Fluids 10, 1 (1997).

[17] P. E. Savage, S. Gopalan, T. I. Mizan, C. J. Martino, and E. E. Brock, AIChE J. 41, 1723 (1995).

[18] J. A. Dahl, B. L. S. Maddux, and J. E. Hutchison, Chem. Rev. 107, 2228 (2007).

[19] C. Song, Catal. Today 115, 2 (2006).

[20] L. Xu, P. Kumar, S. V. Buldyrev, S.-H. Chen, P. H. Poole, F. Sciortino, and H. E. Stanley, Proc. Natl. Acad. Sci. U.S.A. 102, 16558 (2005).

[21] V. V. Brazhkin, Yu. D. Fomin, A. G. Lyapin, V. N. Ryzhov, and K. Trachenko, Phys. Rev. E 85, 031203 (2012).

[22] V. V. Brazhkin, Yu. D. Fomin, A. G. Lyapin, V. N. Ryzhov, E. N. Tsiok, and K. Trachenko, Phys. Rev. Lett. 111, 145901 (2013).

[23] V. V. Brazhkin and K. Trachenko, Phys. Today 65(11), 68 (2012).

[24] C. Yang, V. V. Brazhkin, M. T. Dove, and K. Trachenko, Phys. Rev. E 91, 012112 (2015).

[25] J. Frenkel, Kinetic Theory of Liquids (Dover, Mineola, NY, 1955).

[26] K. Trachenko and V. V. Brazhkin, Rep. Prog. Phys. 79, 016502 (2016).

[27] L. Wang, C. Wang, M. T. Dove, Yu. D. Fomin, V. V. Brazhkin, and K. Trachenko, Phys. Rev. E 95, 032116 (2017).

[28] S. M. Stishov, Pis'ma Zh. Eksp. Teor. Fiz. 57, 189 (1993) [JETP Lett. 57, 196 (1993)].
[29] V. V. Brazhkin, A. G. Lyapin, V. N. Ryzhov, K. Trachenko, Yu. D. Fomin, and E. N. Tiosk, Phys. Usp. 55, 1061 (2012).

[30] A. K. Soper and D. T. Bowron, Chem. Phys. Lett. 683, 529 (2017).

[31] National Institute of Standards and Technology database, see https://webbook.nist.gov/chemistry/fluid.

[32] D. T. Bowron, A. K. Soper, K. Jones, S. Ansell, S. Birch, J. Norris, L. Perrott, D. Riedel, N. J. Rhodes, S. R. Wakefield, A. Botti, M. A. Ricci, F. Grazzi, and M. Zoppi, Rev. Sci. Instrum. 81, 33905 (2010).

[33] A. K. Soper, http://purl.org/net/epubs/work/56240, accessed 14 May 2019.

[34] I. T. Todorov, B. Smith, M. T. Dove, and K. Trachenko, J. Mater. Chem. 16, 1911 (2006).

[35] M. Gao, A. J. Misquitta, C. Yang, I. T. Todorov, A. Mutter, and M. T. Dove, Mol. Syst. Des. Eng. 2, 457 (2017).

[36] Z. Zhang and Z. Duan, J. Chem. Phys. 122, 214507 (2005).

[37] C. Prescher, Yu. D. Fomin, V. B. Prakapenka, J. Stefanski, K. Trachenko, and V. V. Brazhkin, Phys. Rev. B 95, 134114 (2017).

[38] D. Smith, M. A. Hakeem, P. Parisiades, H. E. Maynard-Casely, D. Foster, D. Eden, D. J. Bull, A. R. L. Marshall, A. M. Adawi, R. Howie, A. Sapelkin, V. V. Brazhkin, and J. E. Proctor, Phys. Rev. E 96, 052113 (2017).

[39] C. Cockrell, O. Dicks, V. V. Brazkhin, and K. Trachenko, arXiv: 1905.00747.

[40] J. M. Ziman, Models of Disorder (Cambridge University Press, Cambridge, UK, 1979).

[41] K. Tsuji, Y. Katayama, Y. Morimoto, and O. Shimomura, J. Non-Crystal. Solids 205, 295 (1996).

[42] K. Tsuji and Y. Katayama, J. Phys.: Condens. Matter 15, 6085 (2003).

[43] S. Yamamoto, Y. Ishibashi, Y. Inamura, Y. Katayama, T. Mishina, and J. Nakahara, J. Chem. Phys. 124, 144511 (2006).

[44] J. Krausser, K. H. Samwer, and A. Zaccone, Proc. Natl. Acad. Sci. U.S.A. 112, 13762 (2015).

[45] H. Akaike, IEEE Trans. Auto. Control 19, 716 (1974).

[46] G. Schwarz, Ann. Stat. 6, 461 (1978).

[47] A. A. Maradudin, E. W. Montroll, G. H. Weiss, and I. P. Ipatova, Theory of Lattice Dynamics in the Harmonic Approximation (Academic Press, New York, 1971).

[48] L. Wang, C. Yang, M. T. Dove, V. V. Brazhkin, and K. Trachenko, J. Phys.: Condens. Matt. 31, 225401 (2019).

[49] J. E. Proctor, C. G. Pruteanu, I. Morrison, I. F. Crowe, and J. S. Loveday, J. Phys. Chem. Lett. 10, 6584 (2019).

[50] V. Pipich and D. Schwahn, Phys. Rev. Lett. 120, 145701 (2018).

[51] T. King, S. Butcher, and L. Zalewski, Apocrita - High Performance Computing Cluster for Queen Mary University of London, Zenodo (2017), doi: https://doi.org/10.5281/zenodo. 438045 . 